\title{
THE EFFECT OF CEMENT THICKNESS AND PREPARATION DESIGN ON STRESS LEVEL AND STRESS DISTRIBUTION IN MAXILLARY CENTRAL INCISOR RESTORED BY LAMINATE VENEERS- A FINITE ELEMENT ANALYSIS
}

\author{
Shima Ghasemi', Amir Reza Babaloo², Sahar Negargar³, Sohrab Amini ${ }^{4}$
}

${ }^{1}$ Faculty, Department of Prosthodontics, Faculty of Dentistry, Tabriz University of Medical Sciences, Tabriz, Iran.

${ }^{2}$ Faculty, Department of Prosthodontics, Faculty of Dentistry, Tabriz University of Medical Sciences, Tabriz, Iran.

${ }^{3}$ Dentist, Faculty of Dentistry, Tabriz University of Medical Sciences, Tabriz, Iran.

${ }_{4}^{4}$ Faculty, Department of Periodontics, Faculty of Dentistry, Ardabil University of Medical Sciences, Ardabil, Iran.

ABSTRACT
BACKGROUND
Laminate veneers have been introduced as a successful and reliable technique for treating malformed, yellow, or chipped teeth.
However, mechanically speaking, their success is questionable. The main reason for the failure of laminate veneer treatments is
their fracture. The present study aims at improving the mechanical features of laminate veneers by investigating the factors
affecting the success of this treatment through a finite element analysis (FEA).

\section{MATERIALS AND METHODS}

For assessing the distribution in the laminate veneer of maxillary central incisor under different clinical conditions, first a healthy natural maxillary central incisor was prepared, and it was scanned by a 3D Scanner. Then, Window finish line design was defined virtually in the software; feathered incisal edge and overlapped incisal edge were defined virtually. The scanned tooth was modelled in SOLIDWORKS 2015, and it was then entered into ABAQUS FEA which is an analytical software. Finally, the maximum stress tolerated in each of these conditions as well as the stress level distribution were recorded and described in different points of laminate veneers.

\section{RESULTS}

The present results showed that in comparison with other preparation designs, Window preparation design creates more tension in the teeth. Moreover, according to the findings obtained from the finite element analysis, overlapped incisal edge preparation creates the most tension in porcelain. In all kinds of preparations, the amount of tension imposed on the cement was significantly less than the tension imposed on teeth and porcelain.

\section{CONCLUSION}

It can be concluded that, the preparation, cement thickness, and the angle of the forces applied will significantly change the maximum internal tension of teeth, porcelain, and the related cement.

\section{KEY WORDS}

Finite Element Analysis, Laminate Veneers, Window Preparation

HOW TO CITE THIS ARTICLE: Ghasemi S, Babaloo AR, Negargar S, et al. The effect of cement thickness and preparation design on stress level and stress distribution in maxillary central incisor restored by laminate veneers- a finite element analysis. J. Evolution Med. Dent. Sci. 2019;8(10):712-718, DOI: 10.14260/jemds/2019/157

\section{BACKGROUND}

Over the last two decades, laminate veneers have been introduced as a successful and reliable technique for treating malformed, yellow, or chipped teeth. In terms of beauty and biology, laminate veneers have been always confirmed by dentists. (1, 2)

The success of this technique depends on the conservative shaving, bonding to enamel, beauty, and colour stability.(3) However, the success of laminate veneers is mechanically questionable. The main reason for the failure of laminate veneers treatments is their fracture. However, there are other factors including decay and biological problems that have a lower frequency. (2)

'Financial or Other Competing Interest': None.

Submission 11-01-2019, Peer Review 20-02-2019,

Acceptance 26-02-2019, Published 11-03-2019.

Corresponding Author:

Sahar Negargar

Faculty of Dentistry,

Tabriz University of Medical Sciences, Tabriz, Iran.

E-mail: sahar.negargar@yahoo.com

DOI: $10.14260 /$ jemds/2019/157

\section{(c) $(7)$}

Thus, it seems necessary to conduct studies that aim to improve the mechanical features of the laminate veneers. Given the variety of materials and their geometric complexity, it is hard to assess the mechanical features and stress distribution of teeth and restorations. (4) However, one of the useful and efficient tools in this regard is finite element analysis (FEA) that is known as a reliable tool for predicting the clinical behaviours of restorative and prosthetic materials.(5) In this method, the biomechanical behaviours of restorative and prosthetic material (Under different forces) and the specific physical characteristics of each element are measured.(3)

Although some clinical studies conclude that the kind of preparation does not affect the strength of laminate veneers, $(6-9)$ the findings of some other studies conducted by using FEA have indicated that the kind of dental preparation affects the distribution of the stress imposed on the laminate as well as their strength. ${ }^{9,10)}$ There are three main preparation for placing laminate veneers including; 1. Window preparation (Limited to the labial surface of the tooth without involving the incisal edge), 2. Feathered incisal edge preparation (Covering the labial entire surface of the 
tooth and involving the incisal edge without a palatal development), and 3. Overlapped incisal edge preparation (Covering the labial surface of the tooth and involving the incisal edge with the palatal involvement).(8) In a study conducted by Li et al (2014) by using a 3-D FEA, it was concluded that porcelain veneers with a palatal chamfer preparation design and composite veneers with a But-joint preparation design tolerated more stress. In this study, the models are loaded through applying two angles of force affecting their stress distribution.(3) In another study conducted by Schmidt et al (2010), it was concluded that the porcelain forces with a palatal chamfer preparation design had the capability of withstanding more occlusal forces.(10)

Some other studies have measured the effect of cement by using FEA, and they have concluded that the kind of cement and its thickness affects the long-term success of the restorations, $(11,12)$ and the cement fracture results in microleakage, laminate veneer fracture, and its detachment from the teeth. $(13,14)$ In a study conducted by Kamposiora et al (2010), it was confirmed that glass ionomer cement and resin cement had a greater ability to withstand stress.(13) In another study conducted by Wimmer et al (2013), it was indicated that the kind of cement and its thickness had a significant effect on reducing the stress imposed on the restoration; in comparison to glass ionomer cement, resin cement showed more resistance against occlusal stresses. Moreover, in comparison to150-micron-thick cement, the 50micron-thick cement showed a better resistance.(15) By searching the information sources available, it was made clear that no study has been yet conducted to study the different kinds of laminate veneer preparations with different cements having various thicknesses. Thus, given the abovementioned materials as well as the contradictions found in this regard, the present study aims at assessing the stress distribution in laminate veneers of maxillary central incisor in different clinical conditions through using finite element analysis. It is hoped that the findings of the present study will be effective in resolving the contradictions in this regard and increasing the clinical quality of laminate veneers.

\section{MATERIALS AND METHODS}

The present study is a descriptive cross-sectional study conducted as a computer-based model. Thus, there is no ethical limitation in the present study.

\section{Method}

First, for preparing the 3-D samples, a healthy central incisor of the front upper jaw (maxilla) was prepared. Then, it was defined separately in three dental models in the software; window preparation, feathered incisal edge preparation, and overlapped incisal edge preparation.

\section{Window Preparation}

The preparation of the buccal surface of the tooth is from one millimeter of CEJ up to one millimeter of the incisal edge of the tooth which is 0.5 deep; all finish lines are chamfershaped.(3)

\section{Feathered Incisal Edge Preparation}

The entire buccal surface of the tooth is prepared for 0.5 millimeters, and its and its occlusal gingival area is 1 millimeter apart from the CEJ of the tooth. On the other side, preparation was developed to the incisal edge. It involved the incisal edge, but did not enter the palatal area of the tooth. In the incisal edge, the finish lines were in the shape of buttjoint, and in other parts they were chamfer-shaped.(3)

\section{Overlapped Incisal Edge Preparation}

The entire buccal surface of the tooth is prepared for 0.5 millimeter; its occlusal gingival area is 1 millimeter apart from the CEJ of the tooth. On the other side, preparation was developed to the incisal edge. It shortened the incisal edge for one millimeter, and the rest of one-millimeter preparation entered the palatal part of the tooth. All the finish lines were chamfer-shaped.(3)

The prepared healthy and natural maxillary central incisor underwent a 3-D scan by using a 3-D Scanner (ATOS, Paris, France). The scanned tooth was modelled by using SOLIDWORKS 2015 (SOLIDWORKS, Boston, USA) (Mesh tetrahedral), and it was then inserted into the ABAQUS FEA (ABAQUS, Inc, Rhode Island, USA) which is an analytical software. In this software, enamel, dentin, pulp, and root length have been defined for every tooth based on the average values. Moreover, the periodontal ligament space of 0.2 millimeter and cancellous bone of 0.7 millimeter were restored and were placed around the teeth.(16) Given the pattern of the natural intact tooth, porcelain laminate veneer was modelled for preparation. In each of the models, two thicknesses were defined for the cement space $(25$ and 50 microns). Furthermore, for each of the models defined, the characteristics of a specific cement were considered; Panavia (Kurary dental, Tokyo, Japan).(1) In the next stage, the 50 newton force was applied in two angles of 60 and 125 degrees (the 60-degree force represents the intercuspal movement, and the 125-degree force represents the Proterozoic movement) in the longitudinal axis on the incisal edge of the tooth, (16) so that the stress distribution of the abutment, laminate veneer, and cement will be investigated. It is supposed to conduct complete cement bonding, and the bonding point of cement to laminate, tooth, and PDL connector is a "Tie Type" (The boundary conditions were such that the alveolar bone freedom was limited for 6 degrees).(3) There were as many as 12 models; the forces were applied to the models in two angles. At the end, the maximum stress sustained (In each of the conditions) as well as the stress distribution of different points of laminate veneers were recorded and described. The elasticity module and the Poisson's ration of all elements were determined by using the reference. In this software, the analysis was conducted after defining the mechanical features of the materials, boundary conditions, loading, and element arrangement, and... This analysis obtained was subsequently studied in more details.

\section{The Statistical Analysis of The Data}

Since statistical analysis cannot be conducted in finite element analysis (FEA) studies, there is no statistical analysis in the present study. 


\section{RESULTS}

The FEA of the simulated models are as follows

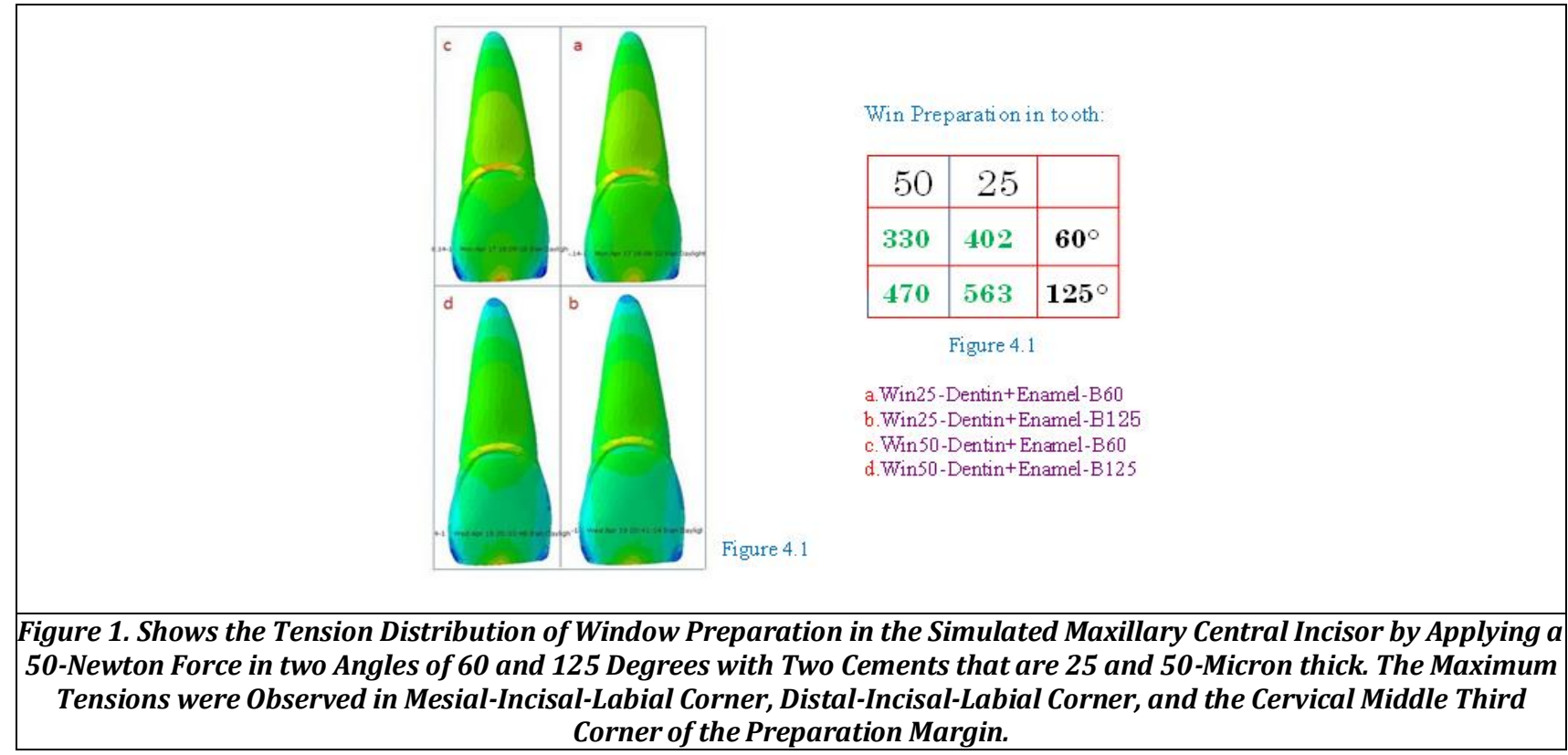

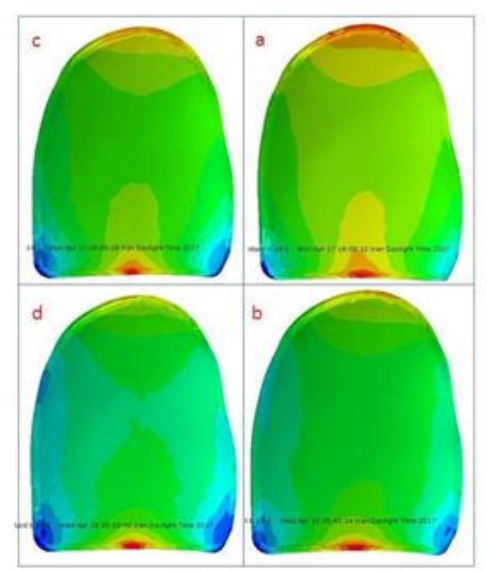

Win Preparation in porcelain:

\begin{tabular}{|l|l|l|}
\hline 50 & 25 & \\
\hline 143 & 109 & $60^{\circ}$ \\
\hline 180 & 173 & $125^{\circ}$ \\
\hline \multicolumn{3}{|c}{ Figure 4.2} \\
\hline
\end{tabular}

a Win25-Ceramics-ling 60

b. Win 25 -Ceramics-ling1 25

c. Win 50 -Ceramics-ling60

d. Win 50-Ceramics-ling1 25

Figure 4.2

Figure 2. Shows the Tension Distribution of Window Preparation in the Related Porcelain of Simulated Maxillary Central Incisor by Applying a 50-Newton Force in Two Angles of 60 and 125 Degrees with Two Cements that are 25 and 50-Micron thick. The Maximum Tensions were Observed in Mid-Incisal Areas, Mesial-Incisal-Labial Corner, Distal-Incisal-Labial Corner, and the Cervical Middle Third Corner of the Preparation Margin.

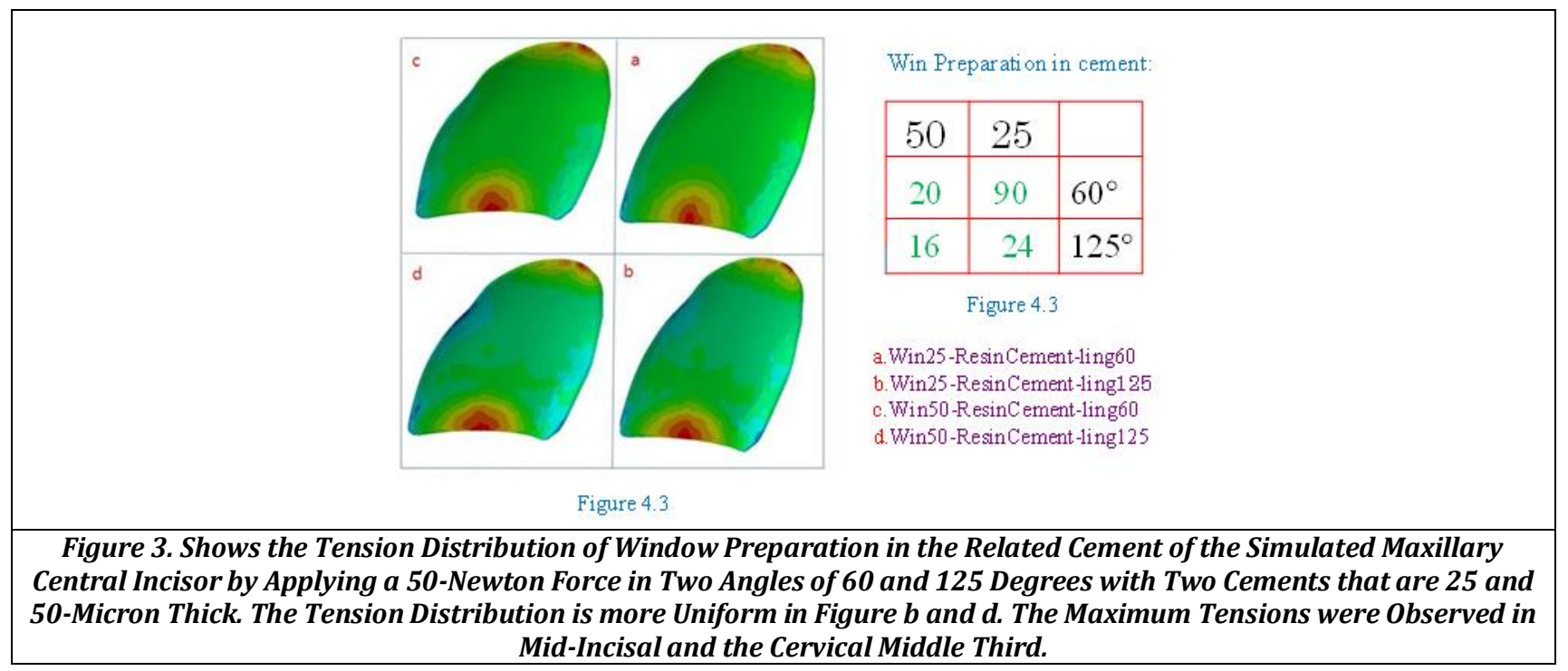




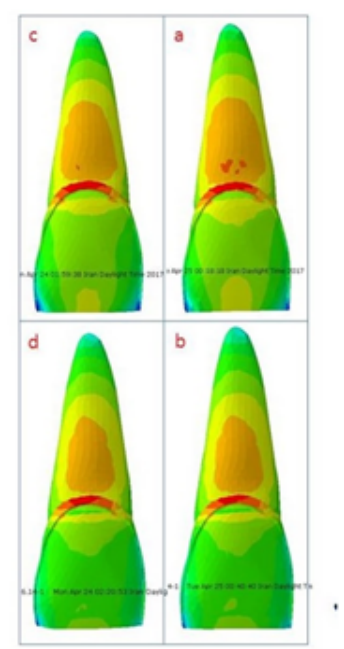

Flat Preparation in tooth:

\begin{tabular}{|l|l|l|}
\hline 50 & 25 & \\
\hline 73 & 72 & $60^{\circ}$ \\
\hline 72 & 72 & $125^{\circ}$ \\
\hline
\end{tabular}

Figure 4.4

a.Flat25-DentintEnamel-B 60

b.Flat25-Dentin+Enamel-B125

c. Flat50-Dentin+Enamel-B 60

d.Flat50-Dentin+Enamel-B 125

Figure 4.4

Figure 4. Shows the Tension Distribution of Feathered Incisal Edge Preparation of the Simulated Maxillary Central Incisor by Applying a 50-Newton Force in Two Angles of 60 and 125 Degrees with Two Cements that are 25 and 50-Micron Thick. The Distribution Pattern of the Tension was like that of Figure 1. However, the Numerical Value of the Maximum Tension is much less than that of Figure 1.
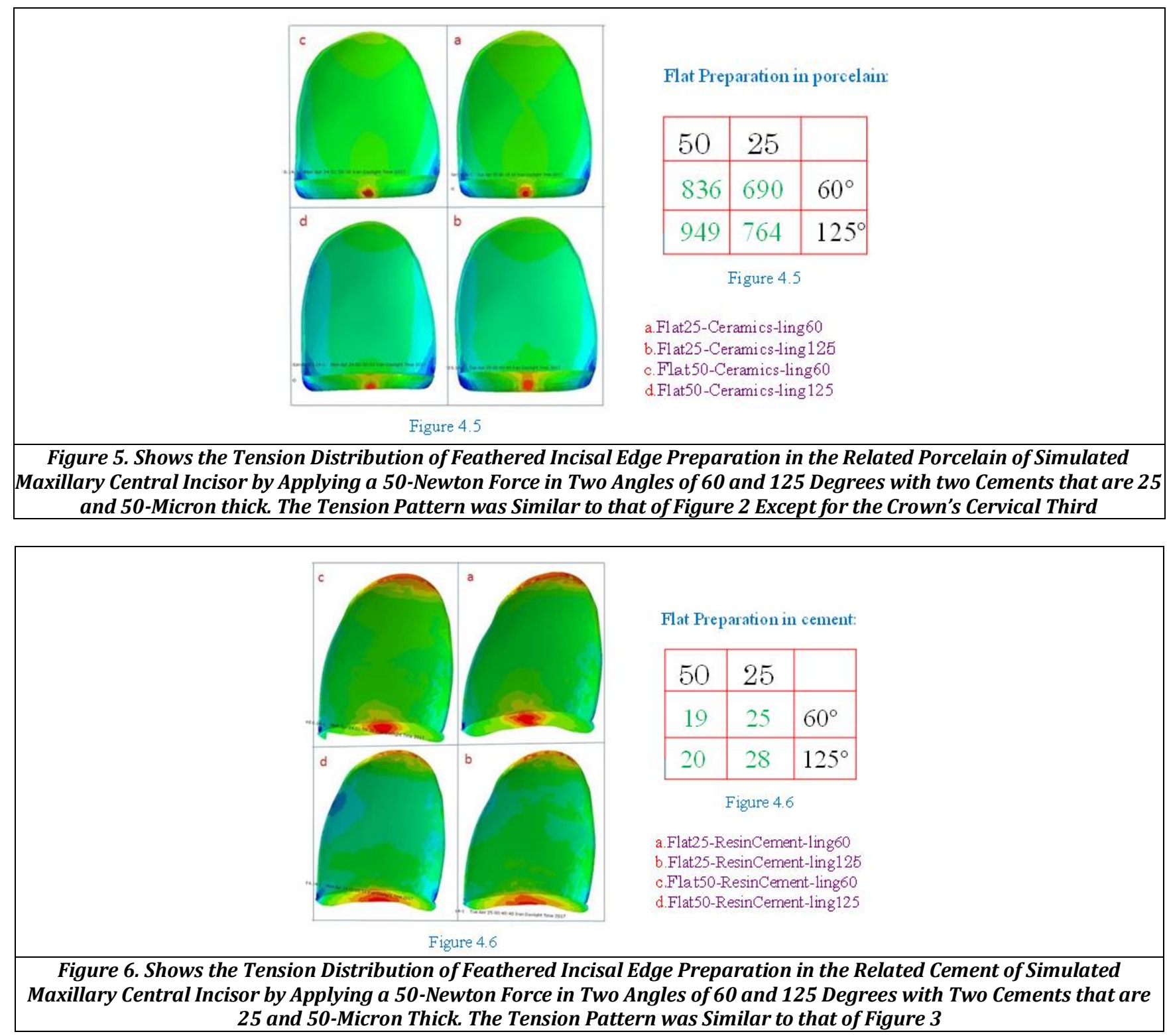


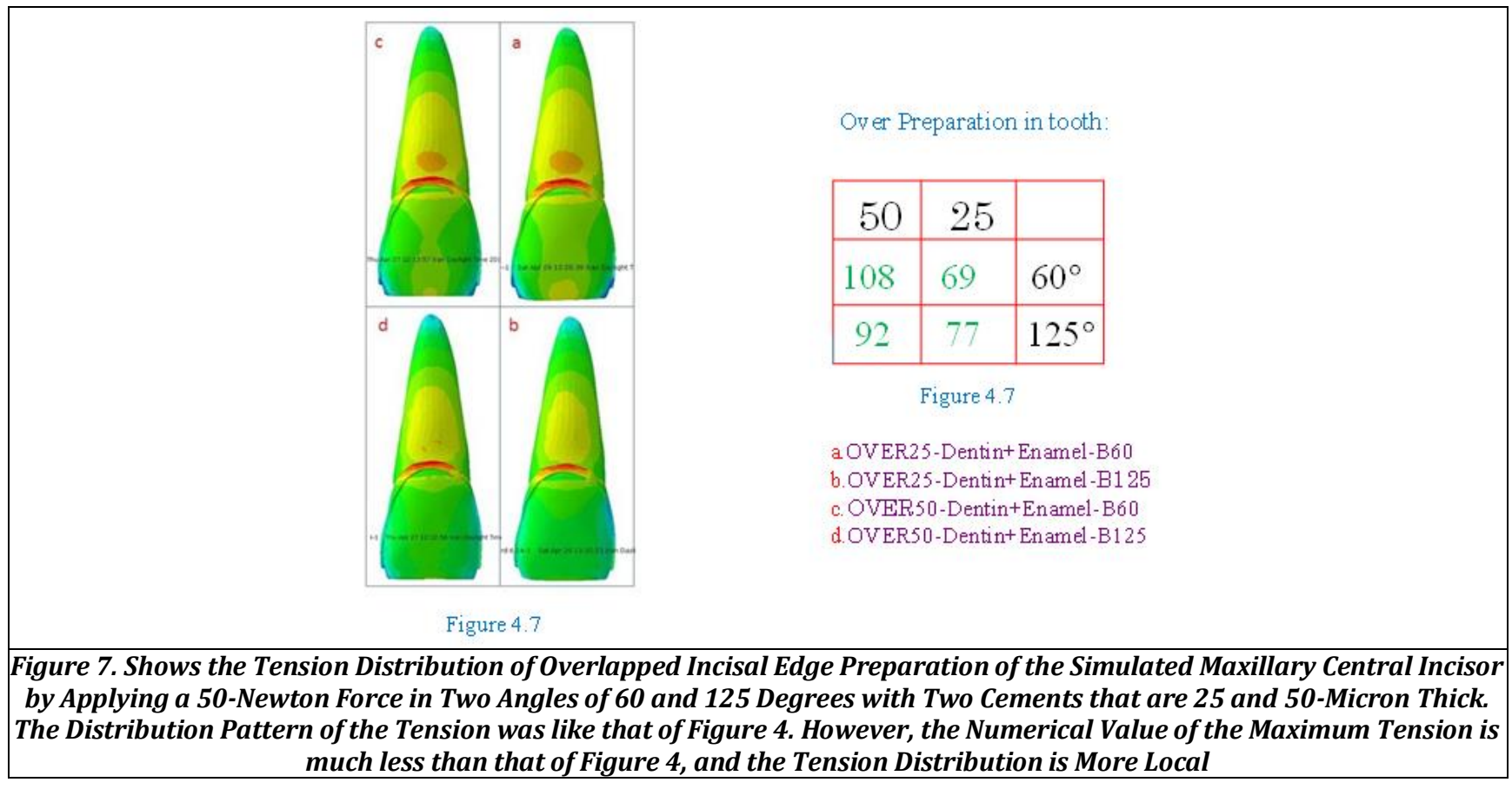

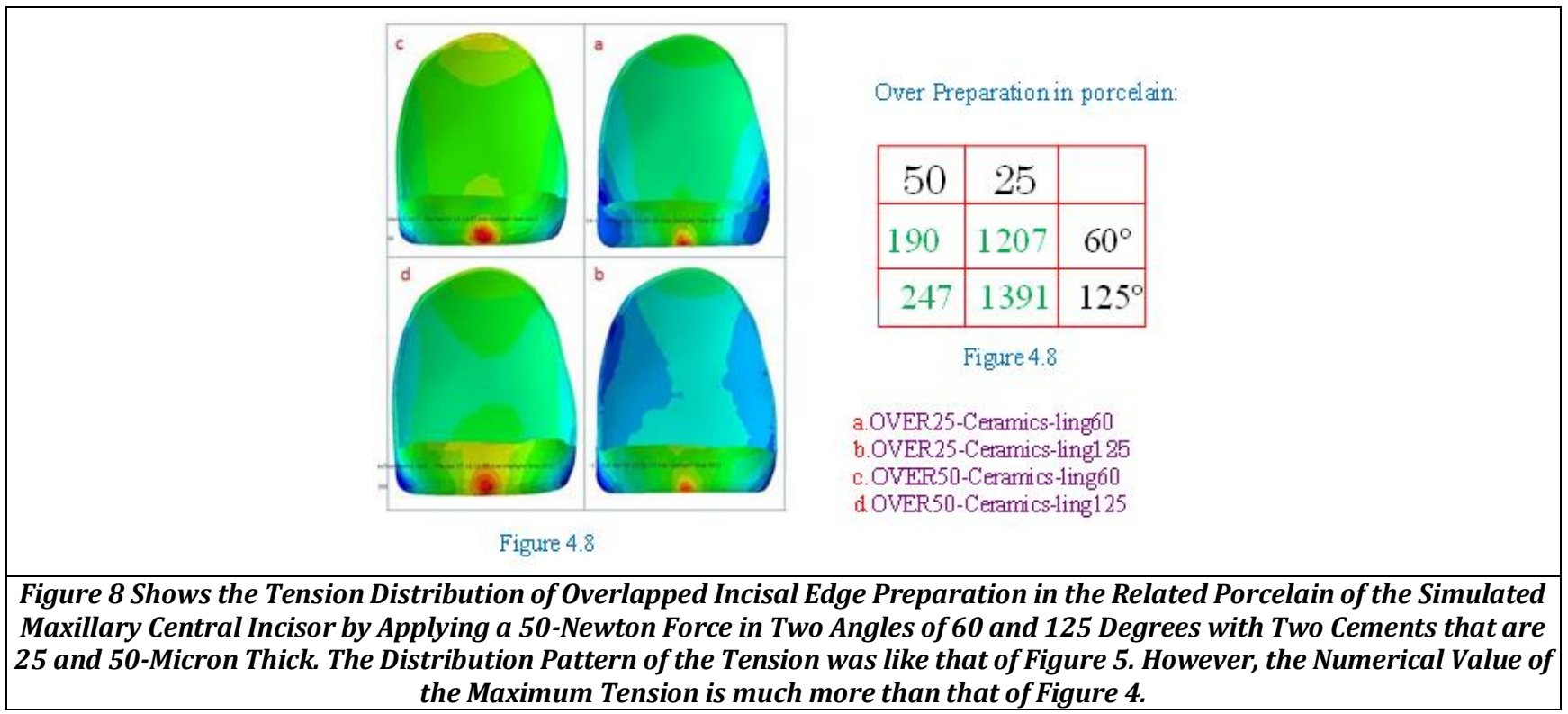

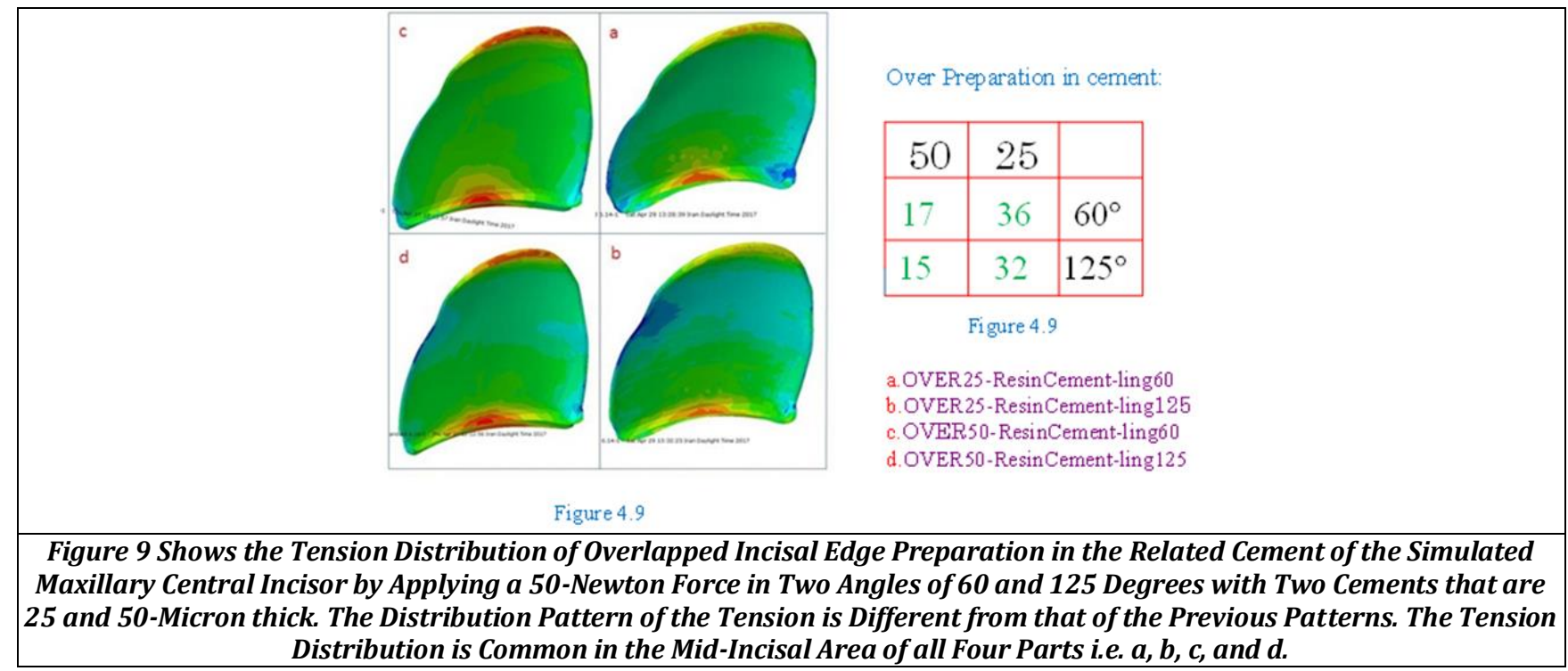




\section{DISCUSSION}

According to the findings obtained from table 1, the maximum dental tension of Window preparation design increased as the angle of the applying force increased, and the tension distribution was more non-uniform in the 125-degree angle than that of 60-degree angle. However, the increase of cement thickness resulted in reduced tension applied.

According to the findings obtained from table 2, the maximum dental tension of porcelain in Window preparation design increased as the angle of the applying force increased, and the tension distribution was more non-uniform in the 125-degree angle than that of 60-degree angle. However, unlike the previous model, the increase of cement thickness resulted in the increased tension applied.

According to the findings obtained from table 3, the maximum dental tension of cement in Window preparation design decreased as the angle of the applying force increased, and the tension distribution was more non-uniform in the 125-degree angle than that of 60-degree angle. Moreover, increased cement thickness resulted in reduced tension applied.

According to the findings obtained from table 4, the maximum dental tension of feathered incisal edge does not affect the tension applied when the angle of applying the force increased. This is true for the increased cement thickness as well.

According to the findings obtained from table 5, the maximum dental tension of porcelain in feathered incisal edge increased when the angle of applying force and cement thickness increased.

According to the findings obtained from table 6, the maximum dental tension of cement in feathered incisal edge increased when the angle of applying force. This is quite reverse as for the increase of cement thickness.

According to the findings obtained from table 7 , the maximum dental tension of overlapped incisal edge preparation decreased with the increased angle of the force applied. However, the tension distribution was more nonuniform in 125-degree angle than that of 60-degree angle. Moreover, increased cement thickness resulted in the reduced tension applied.

According to the findings obtained from table 8, the maximum dental tension of porcelain in the overlapped incisal edge preparation decreased with the increased angle of the force applied as well as the increased cement thickness.

According to the findings obtained from table 9, the maximum dental tension of cement in the overlapped incisal edge preparation decreased with the increased angle of the force applied and the increased cement thickness.

The findings of the present study indicate that Window preparation design results in more tension in tooth than other preparation designs. Furthermore, according to the findings of finite element analysis, overlapped incisal edge preparation design created the maximum tension in porcelain. In all kinds of preparation designs, the stress applied on the cement is significantly less than the stress applied on tooth and porcelain. Moreover, with the increase of force application angle and cement thickness, the tension resulted became more non-uniform and increased.

Wimmer et al (2013) conducted a study by using finite element analysis to investigate the effect of different cement thicknesses and its features on the stress created and its distribution of zirconium fixed prostheses; greater thicknesses result in more stress in zirconium frame. However, this increased thickness result in reduced stress of the cement itself.(17) The increased cement thickness result in reduced stress of the cement itself; the findings of the present study are consistent with that of Wimmer in this regard. However, the increased cement thickness does not have a significant relationship with increased or reduced stress in tooth and porcelain of different preparation designs.

Cotert et al (2009) conducted a clinical study to investigate the effect of different preparation designs on the treatment success of laminate veneers in 40 patients having 200 laminate veneers. The findings of their study indicated that the kind of preparation had a significant effect on the durability of laminate veneers $(\mathrm{P}<0.05)$. Moreover, according to aforementioned study, overlapped incisal edge preparation design had a significant effect on the entire success of the treatment.(18) The findings of the present study conform to those of the study conducted by Cotert et al; both indicate that the preparation design has a significant effect on the durability of laminate veneer. However, the findings of the present study do not conform to those of the study conducted by Cotert et al; they do not agree whether overlapped incisal edge preparation design results in the entire success of the treatment process. According to the findings of the present study, overlapped incisal edge preparation design results in the maximum tension in the porcelain.

\section{CONCLUSION}

The findings of the present study indicate that the preparation design, cement thickness, and the angles of the forces applied result in a significant change in the maximum internal tension of tooth, porcelain, and cement. In comparison to other preparation designs, window preparation design creates more stress in teeth. Furthermore, according to the findings obtained from the finite element analysis, overlapped incisal edge preparation creates the maximum tension in porcelain. In all kinds of preparation designs, the tension applied on the cement is significantly less than the tension imposed on tooth and porcelain. Moreover, with the increase of force application angle and cement thickness, the tension resulted became more non-uniform and increased.

\section{REFERENCES}

[1] Peumans M, Van Meerbeek B, Lambrechts P, et al. Porcelain veneers: a review of the literature. Journal of Dentistry 2000;28(3):163-77.

[2] Guess PC, Stappert CF. Midterm results of a 5-year prospective clinical investigation of extended ceramic veneers. Dental Materials 2008;24(6):804-13.

[3] Li Z, Yang Z, Zuo L, et al. A three-dimensional finite element study on anterior laminate veneers with different incisal preparations. The Journal of Prosthetic Dentistry 2014;112(2):325-33.

[4] Zarone F, Apicella D, Sorrentino R, et al. Influence of tooth preparation design on the stress distribution in maxillary central incisors restored by means of alumina porcelain veneers: a 3D-finite element analysis. Dental Materials 2005;21(12):1178-88. 
[5] Ausiello P, Apicella A, Davidson C, et al. 3D-finite element analyses of cusp movements in a human upper premolar, restored with adhesive resin-based composites. Journal of Biomechanics 2001;34(10):1269-77.

[6] Castelnuovo J, Tjan AH, Phillips K, et al. Fracture load and mode of failure of ceramic veneers with different preparations. The Journal of Prosthetic Dentistry 2000;83(2):171-80.

[7] Hui KK, Williams B, Davis EH, et al. A comparative assessment of the strengths of porcelain veneers for incisor teeth dependent on their design characteristics. British Dental Journal 1991;171(2):51-5.

[8] Zarone F, Epifania E, Leone G, et al. Dynamometric assessment of the mechanical resistance of porcelain veneers related to tooth preparation: a comparison between two techniques. The Journal of Prosthetic Dentistry 2006;95(5):354-63.

[9] Stappert CF, Ozden U, Gerds T, et al. Longevity and failure load of ceramic veneers with different preparation designs after exposure to masticatory simulation. The Journal of Prosthetic Dentistry 2005;94(2):132-9.

[10] Schmidt KK, Chiayabutr Y, Phillips KM, et al. Influence of preparation design and existing condition of tooth structure on load to failure of ceramic laminate veneers. The Journal of Prosthetic Dentistry 2011;105(6):374-82.

[11] Shahrbaf S, Van Noort R, Mirzakouchaki B, et al. Effect of the crown design and interface lute parameters on the stress-state of a machined crown-tooth system: a finite element analysis. Dental Materials 2013;29(8):e123-31.
[12] Addison 0, Fleming GJ. The influence of cement lute, thermocycling and surface preparation on the strength of a porcelain laminate veneering material. Dental Materials 2004;20(3):286-92.

[13] Kamposiora P, Papavasiliou G, Bayne SC, et al. Predictions of cement microfracture under crowns using 3D-FEA. Journal of Prosthodontics 2000;9(4):201-9.

[14] Silva NR, Calamia CS, Harsono M, et al. Bond angle effects on microtensile bonds: laboratory and FEA comparison. Dental Materials 2006;22(4):314-24.

[15] Wimmer T, Erdelt KJ, Raith S, et al. Effects of differing thickness and mechanical properties of cement on the stress levels and distributions in a three-unit zirconia fixed prosthesis by FEA. Journal of Prosthodontics 2014;23(5):358-66.

[16] Fradeani M, Redemagani M, Corrado M. Porcelain laminate veneers: 6-12 year clinical evaluation-a retrospective study. Int J Periodontics Restorative Dent 2005;25(1):9-17.

[17] Wimmer T, Erdelt KJ, Raith S, et al. Effects of differing thickness and mechanical properties of cement on the stress levels and distributions in a three-unit zirconia fixed prosthesis by FEA. Journal of Prosthodontics 2014;23(5):358-66.

[18] Cotert HS, Dündar M, Oztürk B. The effect of various preparation designs on the survival of porcelain laminate veneers. J Adhes Dent 2009;11(5):405-11. 